\title{
External Limiting Membrane, Photoreceptor Ellipsoid Zone Disruption, and Retinal Pigment Epithelium Alterations in Diabetic Retinopathy
}

\author{
Nibha Mishra ${ }^{1}$ Gurkiran Kaur ${ }^{1}$ Sandeep Saxena ${ }^{1}$ \\ ${ }^{1}$ Department of Ophthalmology, King George's Medical University, \\ Lucknow, Uttar Pradesh, India \\ Address for correspondence Sandeep Saxena, MS, FAMS, FRCS, \\ Department of Ophthalmology, King George's Medical University, \\ Lucknow 226003, Uttar Pradesh, India \\ Ann Natl Acad Med Sci (India) 2022;58:119-124. \\ (e-mail: sandeepsaxena2020@yahoo.com).
}

\begin{abstract}
Keywords

- diabetic retinopathy

- ellipsoid zone

- external limiting membrane

- retinal pigment epithelium

- spectral domain optical coherence tomography

Objective Diabetic retinopathy (DR), a microvascular complication of diabetes, is a leading cause of preventable blindness. Spectral domain optical coherence tomography (SD-OCT) provides cross-sectional and topographical imaging of the retina. SDOCT resolves outer retinal layers into three hyperreflective bands-external limiting membrane (ELM), ellipsoid zone (EZ), and retinal pigment epithelium (RPE). In this article, we have studied the role of these outer retinal layers in structural and molecular changes taking place in DR.

Materials and Methods Articles with clinical features, pathogenesis, diagnosis, and treatment of DR were thoroughly studied. Articles were searched on PubMed, MEDLINE, and Cochrane Library from 2000 to 2020. Studies focusing on the role of ELM, EZ, and RPE in pathogenesis of DR based on SD-OCT were included.

Results The long-standing hyperglycemia leads to protein glycosylation resulting in formation of advanced glycation end products (AGEs). AGEs have an impact through their effect on retinal microvasculature, vascular endothelial growth factor (VEGF), intercellular adhesion molecule-1, nitrosative and oxidative stress, and vitamin D and calcium metabolism. All these factors have been linked with disruption of outer retinal layers. AGEs lead to vascular endothelial dysfunction and release of proangiogenic factors by increasing the expression of VEGF in retinal pericytes and RPE cells. This leads to leakage and fluid accumulation resulting in diabetic macular edema (DME). In DME, there is sequential disruption of ELM and EZ and decrease in visual acuity (VA). The RPE alterations have been reported to be associated with the severity of DR and decrease in VA. Anti-VEGF therapy, most common treatment of DME, leads to restoration of barrier effect of ELM, it was found to be restored first followed by EZ restoration. Newer anti-AGEs agents and their receptor blockers are being developed which have a positive effect on maintaining the health of RPE. Conclusion A complex molecular association exists between the structural changes in ELM, EZ, and RPE in DR. SD-OCT is an indispensable tool to study these changes as integrity of these outer layers of retina is essential for maintaining visual function of retina in DR.
\end{abstract}

published online February 16, 2022
DOI https://doi.org/

$10.1055 / \mathrm{s}-0042-1742585$. ISSN 0379-038X.
(C) 2022. National Academy of Medical Sciences (India). All rights reserved.

This is an open access article published by Thieme under the terms of the Creative Commons Attribution-NonDerivative-NonCommercial-License, permitting copying and reproduction so long as the original work is given appropriate credit. Contents may not be used for commercial purposes, or adapted, remixed, transformed or built upon. (https://creativecommons.org/ licenses/by-nc-nd/4.0/)

Thieme Medical and Scientific Publishers Pvt. Ltd., A-12, 2nd Floor, Sector 2, Noida-201301 UP, India 


\section{Introduction}

Diabetes has become a global burden and the prevalence rates are rising steeply in developing economies. ${ }^{1}$ Diabetes is a progressive disorder leading to complications divided into small vessel or microvascular disease and large vessel or macrovascular disease. Microvascular complications affect the innermost layer of the eye-the retina known as diabetic retinopathy (DR). All individuals with diabetes are at risk of developing DR. Understanding of its pathogenesis is essential for developing more effective modalities for treatment. DR is broadly divided into nonproliferative with dot-blot hemorrhages, cotton-wool spots, venous beading or intraretinal microvascular anomalies, and proliferative retinopathy defined by the presence of neovascularization of the optic disc or elsewhere in other parts of

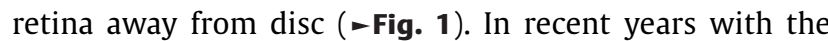
advent of advanced retinal imaging techniques, the understanding of different retinal disorders have become easier. Spectral domain optical coherence tomography (SD-OCT) has emerged as an indispensable tool for in vivo retinal imaging providing layer by layer detailed analysis of retina. Outer retinal layers comprising external limiting membrane (ELM), photoreceptor ellipsoid zone (EZ), and retinal pigment epithelium (RPE) have a very important role to play in different retinal disorders, and their integrity is essential for maintaining visual function of the eye. ${ }^{2-5}$ In this article, we have described the role of these outer layers of retina in DR based on SD-OCT.

\section{Materials and Methods}

Articles with clinical features, pathogenesis, diagnosis, and treatment of DR were thoroughly studied. Articles were searched on PubMed, MEDLINE, and Cochrane Library from 2000 to 2020. Studies focusing on the role of ELM, EZ, and RPE in the pathogenesis of DR based on SD-OCT were included.

\section{Spectral Domain Optical Coherence Tomography: Role in the Assessment of Retinal Morphology}

Optical coherence tomography (OCT) is a noninvasive imaging modality that provides cross-sectional and topographical imaging of the posterior segment of eye (-Fig. 2). OCT provides ophthalmologists to have a nearly cellular level of resolution of the retina. It utilizes light to image tissue using low coherence interferometry. SD-OCT is currently the most widely used OCT technology. It uses 820 - to 880 -nm probing light with a scan rate of $52,000 \mathrm{~Hz}$ or greater providing excellent imaging of all the layers of retina. ${ }^{6}$ Human retina has 10 layers. On OCT cross-sectional imaging, they are broadly classified into inner retinal layers and outer retinal layers. SD-OCT resolves mainly three bands in the outer retina as hyperreflective bands which are of great importance as they are responsible for the retinal functions (-Fig. 3A)

\section{Outer Layers of Retina}

\section{External Limiting Membrane}

The innermost hyperreflective band is the ELM, a linear confluence of junctional complexes between Muller cells and photoreceptors. ${ }^{7}$ This band typically is thinner and much fainter than the others. The ELM separates the layers of rods and cones from the overlying outer nuclear layer, and is a linear confluence of junctional complexes between Muller cells and photoreceptors. It serves as a barrier against macromolecules.

\section{Ellipsoid Zone}

The second band is the boundary between inner and outer segments of the photoreceptors, aligned with the ellipsoid portion of the inner segments, and is termed as EZ. ${ }^{8}$ The photoreceptors include an outer segment that absorbs light and converts it into electrical signals and an inner segment

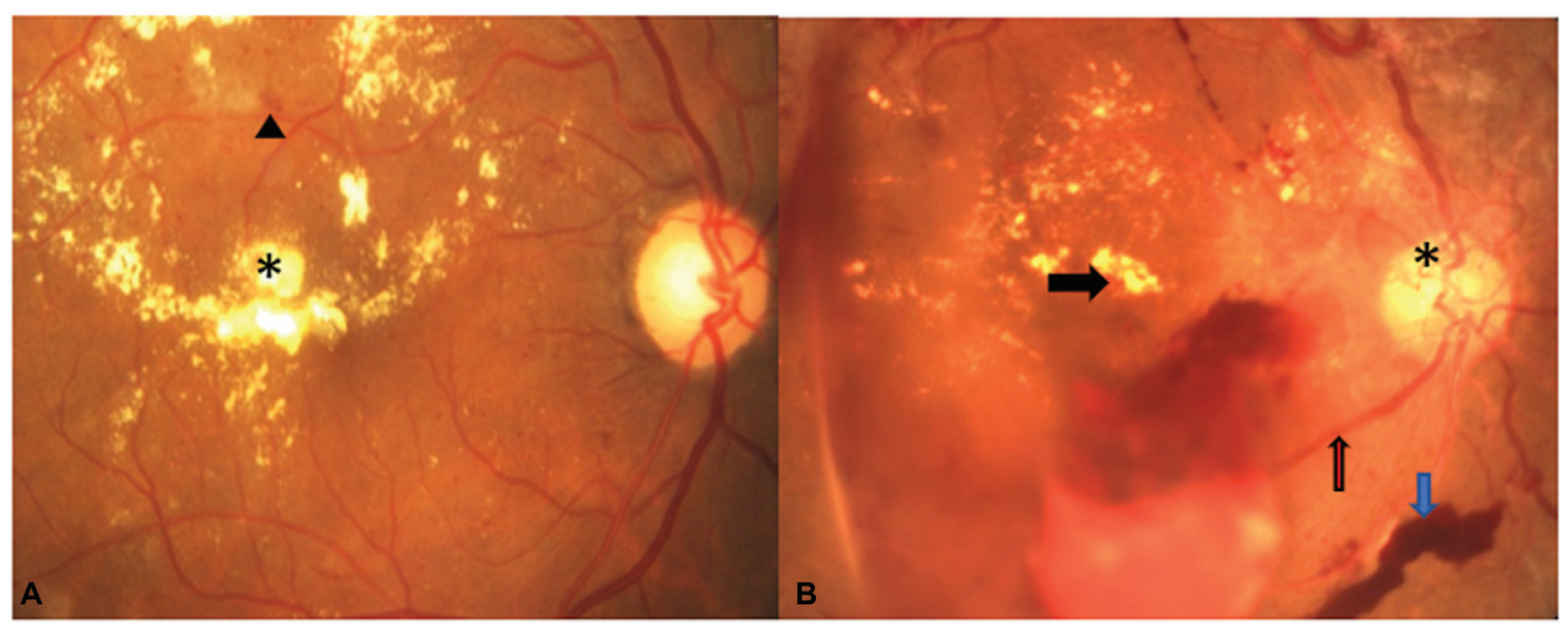

Fig. 1 (A) Retinal exudates (asterisk) and retinal hemorrhages (triangle), features of nonproliferative diabetic retinopathy. (B) Preretinal hemorrhages (blue arrow), hard exudates (black arrow), venous beading (red arrow), and neovascularization of disc (asterisk), all features of proliferative diabetic retinopathy. 


\section{\begin{tabular}{ll|l|l}
\hline Macula Thickness : Macular Cube 512x128 & OD $\bigcirc$ & $\bigcirc$ & OS \\
\hline
\end{tabular}}

A

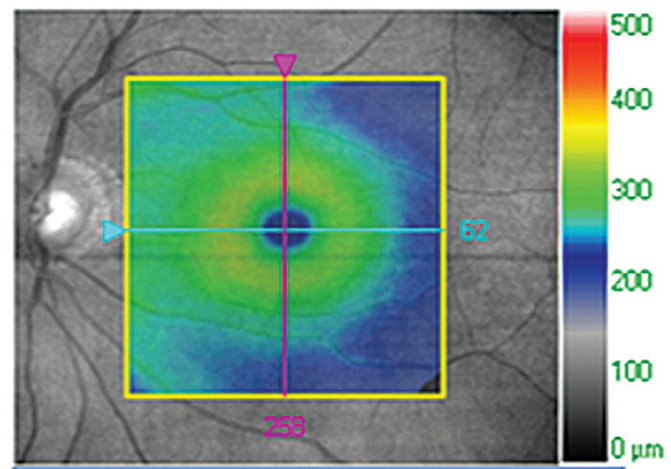

Overlay: ILPS - RPE Transparency. $50 \%$
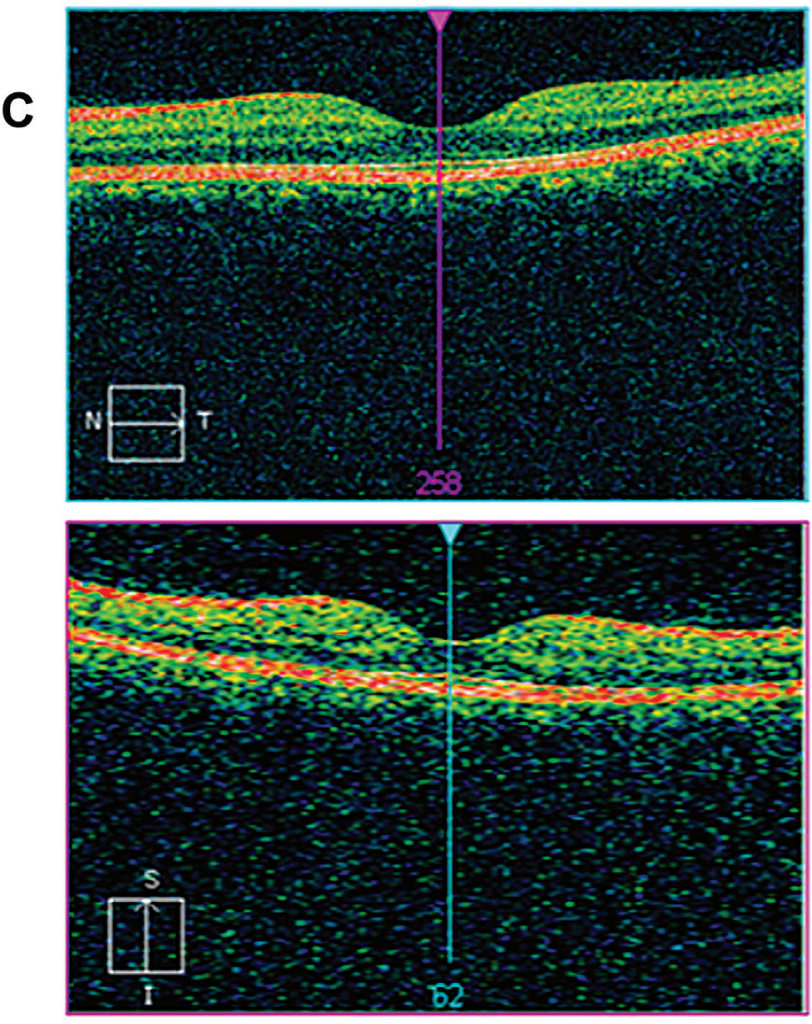

B

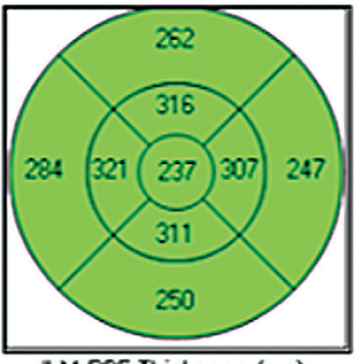

LM-fore Thidkness (um)

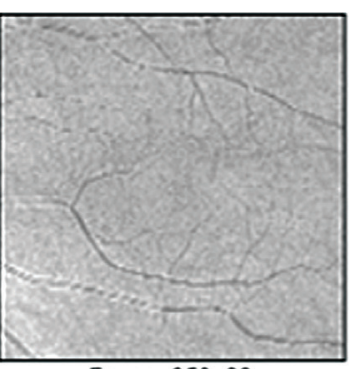

Fovea 258,62

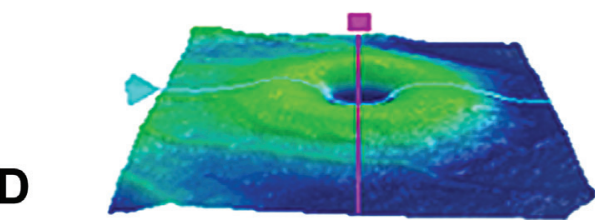

LWM - ROE

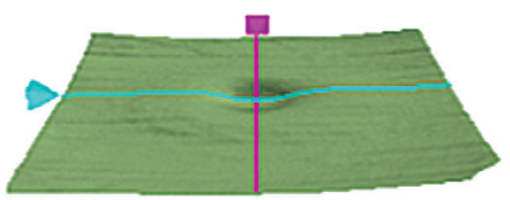

I.M.

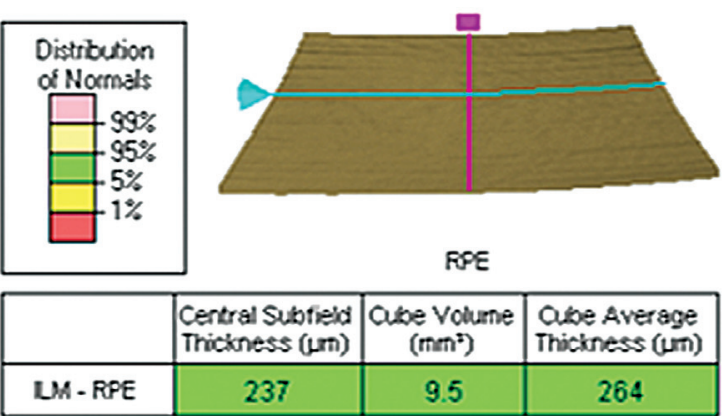

Fig. 2 Macular analysis (macular cube $512 \times 128$ ) done on spectral domain optical coherence tomography. (A) The scanning laser ophthalmoscopy image of the fundus with superimposed color-coded retinal thickness map. (B) The Early Treatment of Diabetic Retinopathy Study grid with values of the retinal thickness $(\mu \mathrm{m})$ in various quadrants. (C) The cross-sectional images of retinal layers. (D) The topographical image of retinal layers.

that has the metabolic functions of generating energy and proteins.

\section{Retinal Pigment Epithelium}

The outermost highly reflective band represents the RPE, Bruch's membrane, and possibly the choriocapillaris. ${ }^{9,10}$ It is the pigmented cell layer just outside the neurosensory retina that nourishes retinal visual cells, and is firmly attached to the underlying choroid and overlying retinal visual cells. The RPE is composed of a single layer of hexagonal cells that are densely packed with pigment granules. The RPE has several functions, namely, light absorption, epithelial transport, spatial ion buffering, visual cycle, phagocytosis, secretion, and immune modulation.

Status of ELM and EZ has been studied in brown Norwegian rats on OCT. It was found that the EZ and ELM disappeared after euthanasia. The origin of the EZ and ELM was found to be related to the biological activities of the photoreceptor cells. ${ }^{11}$

\section{Role of Outer Layers of Retina in Pathogenesis of Diabetic Retinopathy}

Pathogenesis of DR is complex involving multiple molecular pathways occurring due to deranged metabolism in DM. The 


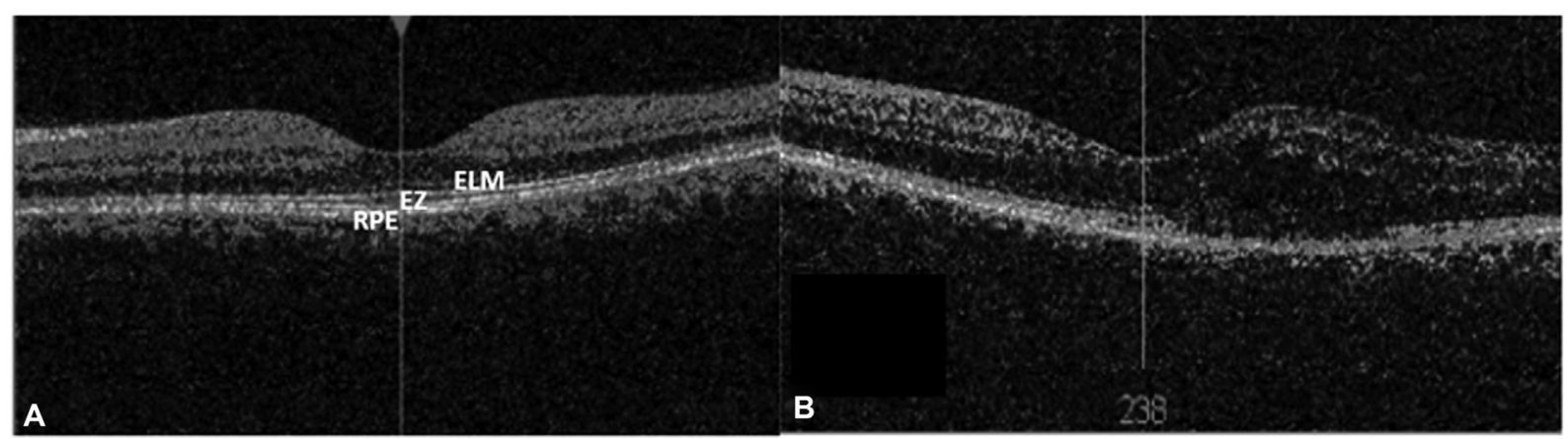

Fig. 3 (A) A cross-sectional spectral domain optical coherence tomography (OCT) image showing hyperreflective outer retinal layer bandsexternal limiting membrane (ELM), ellipsoid zone (EZ), and retinal pigment epithelium (RPE). (B) Spectral domain-OCT cross-sectional image of retina showing disruption of EZ and ELM in a patient of diabetic macular edema.

long-standing hyperglycemia leads to protein glycosylation resulting in the formation of AGEs. AGEs have an impact through their effect on retinal microvasculature, vascular endothelial growth factor (VEGF), intercellular adhesion molecule-1 (ICAM-1), nitrosative and oxidative stress, and vitamin D and calcium metabolism.

AGEs and their receptor activation leads to endothelial dysfunction and release of proangiogenic factors resulting in pericyte apoptosis, vascular inflammation, and angiogenesis damaging the vascular components of the retina. ${ }^{12,13}$ This leads to leakage and fluid accumulation resulting in macular edema. AGEs increase the expression of VEGF in retinal pericytes and RPE cells. ${ }^{14}$ Healthy retina requires interaction between its photoreceptors and the RPE. An intact ELM has been highlighted as a prerequisite for an intact EZ. ${ }^{15}$ Diabetic macular edema (DME) is known to be associated with ELM and EZ disruption (-Fig. 3B). ${ }^{16,17}$

ELM comprises attachment of outer process of glial Muller cells to one another and also to inner photoreceptor segments which has been demonstrated in animal model. ${ }^{18}$ They revealed that tight junctions existed in the ELM between glial Muller cells and photoreceptors. Occludin was found as a key component of tight junctions. In ELM, occludin was found to be organized between the glial Muller cells and the photoreceptors, thus the ELM should be considered as part of a retinal barrier. In DME, glial Muller cells at the level of ELM are swollen and lose their occludin content. ELM junctions thus could be considered as unique regulatory targets in treatment. VEGF alters tight junctions and promotes vascular permeability in many retinal diseases. Murakami et al highlighted the role of occludin in regulation of endothelial barrier properties. $^{19}$

The EZ serves as a clinical indicator of photoreceptor integrity. Biologically, EZ consists of mitochondria mainly enabling higher levels of energy consumption within the photoreceptors. ${ }^{20}$ Mitochondrial dysfunction occurs in the photoreceptors at fovea in DME. The absence of the subfoveal EZ on SD-OCT corresponds to the reduced reflectivity or anatomic absence of the $\mathrm{EZ}$.

Studies demonstrating early retinal damage in experimental diabetes have observed morphological changes in RPE. $^{21}$ Oxidative stress has been found to result in lipid peroxidation and oxidation of glycated proteins in the diabetic retina. ${ }^{22}$ An association of nitrosative and oxidative stress with retinal photoreceptor EZ and topographical changes in RPE has been demonstrated (-Fig. 4). ${ }^{23}$ Experimental studies have shown interaction of RPE and endothelial cells results in loss of barrier function of RPE which was mediated by VEGF. The balance between VEGF and antiangiogenic factor pigment epithelium-derived factor secreted
A

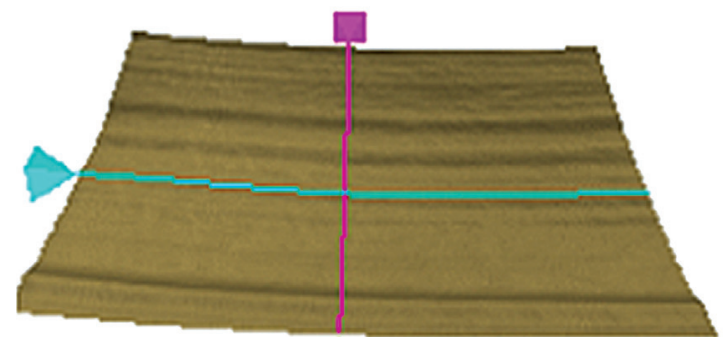

B

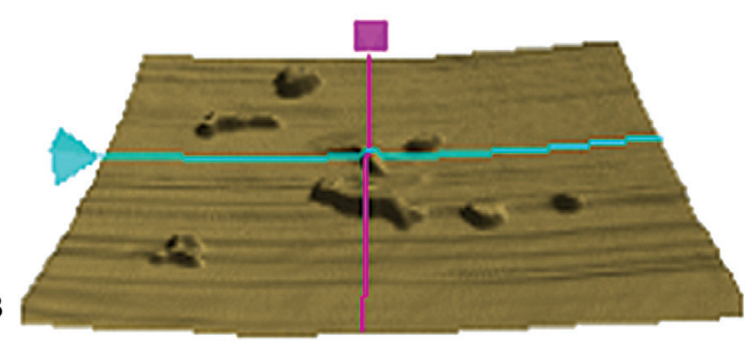

Fig. 4 (A) A normal single-layer retinal pigment epithelium (RPE) map on spectral domain optical coherence tomography (SD-OCT). It is used to get a three-dimensional topographical view of the RPE layer. (B) The single-layer RPE map on SD-OCT of a patient with diabetic retinopathy shows numerous RPE alterations. 
by RPE is crucial for progression of DR. ${ }^{24}$ The VEGF secreted by RPE is essential for maintaining the structural integrity of the outer retina and choriocapillaris.

Retinal VEGF induces ICAM-1 expression leading to leukostasis and breakdown of blood-retinal barrier. ${ }^{25}$ Jain et al demonstrated the correlation of VEGF and ICAM-1 on ELM and EZ disruption ${ }^{15}$ (- Fig. 4). AGEs trigger oxidative stress generation resulting in inflammatory and thrombogenic reactions. Mitochondrial superoxide generation plays an important role in the formation and accumulation of AGEs under diabetic conditions. ${ }^{26}$ Sharma et al showed nitric oxide and oxidative stress in DR are associated with disruption of ELM and EZ. ${ }^{23}$ Increased serum antimyeloperoxidase antibody levels have been found to be associated with ELM and EZ disruption in DR. ${ }^{27}$ Deranged metabolism in DM leads to compromised nutritional status. Vitamin D deficiency has been found to be associated with disruption ELM and EZ. ${ }^{28}$ Vitamin D also plays an important role in regulating calcium homeostasis. Increased serum ionized calcium induces retinal photoreceptor apoptosis resulting in ELM and EZ disruption in DR. ${ }^{29}$

\section{Effect of Outer Layers of Retina on Visual Function in Diabetic Retinopathy}

The ELM and EZ integrity are essential for the maintenance of normal vision. Many cross-sectional or longitudinal studies have shown the clinical importance of both the layers in DME. The length of the disrupted or absent EZ has been associated with visual impairment. ${ }^{30}$ DME is known to be associated with disruption of ELM and EZ, which in turn affects visual acuity (VA). An increase in VEGF and ICAM-1 levels correlates with increased macular thickness (central subfield thickness), sequential disruption of ELM and EZ, and an increase in severity of DR..$^{15}$ Accordingly, disruption of ELM and EZ has been graded as per our published classification: grade 0: no disruption of ELM and EZ; grade 1: ELM disrupted, EZ intact; and grade 2: both ELM and EZ disrupted. This a physician-friendly grading system with an excellent reproducibility and is an important predictor of disease severity and visual outcome. EZ disruption was found to be significantly associated with the severity of retinopathy. RPE which forms the outer retinal barrier in turn maintains nutrition and normal physiology of photoreceptors and inner retinal layers. The RPE alterations have been reported to be associated with the severity of DR and decrease in VA $\left(-\right.$ Fig. 4B). ${ }^{31}$

\section{Effects of Therapeutic Agents of Diabetic Retinopathy on Outer Layers of Retina}

Anti-VEGFs are considered as the first-line treatment for DME. Administration of intravitreal anti-VEGF agents has been found to be associated with improvement in VA. Restoration of the foveal photoreceptors occurs following administration of intravitreal ranibizumab, a Food and Drug Administration-approved anti-VEGF agent in DME has been reported. ${ }^{20}$ Improvement in photoreceptor integrity takes place after second and third doses of ranibizumab with improvement in VA and color vision. A larger foveal photoreceptor microstructure defect is associated with lower VA. Patients with larger foveal photoreceptor microstructure defects at baseline had lesser VA improvements. ${ }^{32}$ The improvement in EZ defect size is dependent on the pattern of DME on SD-OCT. ${ }^{33}$

De et al discovered the mechanism of ELM and EZ restoration after anti-VEGF therapy in DME. ${ }^{34}$ Anti-VEGF therapy led to restoration of barrier effect of ELM. The ELM was established as a retinal structural barrier and was found to restore first followed by EZ restoration. Decrease in logMAR VA was more pronounced in patients associated with restoration of ELM and EZ.

An increase in VEGF results in sequential ELM and EZ disruption on SD-OCT. An intact ELM is a prerequisite for an intact EZ in DME. Anti-VEGF therapy leads to restoration of barrier effect of ELM. The ELM restores first, followed by EZ restoration.

Newer anti-AGEs agents and their receptor blockers are being developed which have a positive effect on maintaining the health of RPE. ${ }^{35}$

\section{Conclusion}

In DR, to understand the complex disease process, the knowledge of outer layers of retina is essential. They play an important role in pathogenesis of DR at both structural and molecular levels. Thus, the integrity of ELM, EZ, and RPE is essential for maintaining visual function of retina in DR.

\section{Author Contribution}

N.M. is the primary author and editor; G.K. compiled the data; and SS is the chief supervisor.

Conflict of Interest

None declared.

\section{References}

1 Pradeepa R, Mohan V. Prevalence of type 2 diabetes and its complications in India and economic costs to the nation. Eur J Clin Nutr 2017;71(07):816-824

2 Shin HJ, Chung H, Kim HC. Association between integrity of foveal photoreceptor layer and visual outcome in retinal vein occlusion. Acta Ophthalmol 2011;89(01):e35-e40

3 Pappuru RR, Ouyang Y, Nittala MG, et al. Relationship between outer retinal thickness substructures and visual acuity in eyes with dry age-related macular degeneration. Invest Ophthalmol Vis Sci 2011;52(09):6743-6748

4 Mitamura Y, Hirano K, Baba T, Yamamoto S. Correlation of visual recovery with presence of photoreceptor inner/outer segment junction in optical coherence images after epiretinal membrane surgery. Br J Ophthalmol 2009;93(02):171-175

5 Kawashima H, Mizukawa K, Watanabe I, Kamao H, Kiryu J. [Evaluation of recovery process of photoreceptor outer segment after retinal detachment repair]. Nippon Ganka Gakkai Zasshi 2011;115(04):374-381

6 Barteselli G, Bartsch DU, Weinreb RN, et al. REAL-TIME FULLDEPTH VISUALIZATION OF POSTERIOR OCULAR STRUCTURES: comparison between full-depth imaging spectral domain optical 
coherence tomography and swept-source optical coherence tomography. Retina 2016;36(06):1153-1161

7 Drexler W, Sattmann H, Hermann B, et al. Enhanced visualization of macular pathology with the use of ultrahigh-resolution optical coherence tomography. Arch Ophthalmol 2003;121(05):695-706

8 Srinivasan VJ, Ko TH, Wojtkowski M, et al. Noninvasive volumetric imaging and morphometry of the rodent retina with high-speed, ultrahigh-resolution optical coherence tomography. Invest Ophthalmol Vis Sci 2006;47(12):5522-5528

9 Puche N, Querques G, Benhamou N, et al. High-resolution spectral domain optical coherence tomography features in adult onset foveomacular vitelliform dystrophy. Br J Ophthalmol 2010;94 (09):1190-1196

10 Gloesmann M, Hermann B, Schubert C, Sattmann H, Ahnelt PK, Drexler W. Histologic correlation of pig retina radial stratification with ultrahigh-resolution optical coherence tomography. Invest Ophthalmol Vis Sci 2003;44(04):1696-1703

11 Yamauchi Y, Yagi H, Usui Y, et al. Biological activity is the likely origin of the intersection between the photoreceptor inner and outer segments of the rat retina as determined by optical coherence tomography. Clin Ophthalmol 2011;5:1649-1653

12 Kollias AN, Ulbig MW. Diabetic retinopathy: early diagnosis and effective treatment. Dtsch Arztebl Int 2010;107(05):75-83

$13 \mathrm{Xu} \mathrm{J}$, Chen LJ, Yu J, et al. Involvement of advanced glycation end products in the pathogenesis of diabetic retinopathy. Cell Physiol Biochem 2018;48(02):705-717

14 Yamagishi S, Amano S, Inagaki Y, et al. Advanced glycation end products-induced apoptosis and overexpression of vascular endothelial growth factor in bovine retinal pericytes. Biochem Biophys Res Commun 2002;290(03):973-978

15 Jain A, Saxena S, Khanna VK, Shukla RK, Meyer CH. Status of serum VEGF and ICAM-1 and its association with external limiting membrane and inner segment-outer segment junction disruption in type 2 diabetes mellitus. Mol Vis 2013;19:1760-1768

16 Sharma SR, Saxena S, Mishra N, et al. The association of grades of photoreceptor inner segment-ellipsoid band disruption with severity of retinopathy in type 2 diabetes mellitus. J Case Rep Stud. 2014;2:502-508

17 Saxena S, Ruia S, Prasad S, et al. Increased serum levels of urea and creatinine are surrogate markers for disruption of retinal photoreceptor external limiting membrane and inner segment ellipsoid zone in type 2 diabetes mellitus. Retina 2017;37(02):344-349

18 Omri S, Omri B, Savoldelli M, et al. The outer limiting membrane (OLM) revisited: clinical implications. Clin Ophthalmol 2010; 4:183-195

19 Murakami T, Felinski EA, Antonetti DA. Occludin phosphorylation and ubiquitination regulate tight junction trafficking and vascular endothelial growth factor-induced permeability. J Biol Chem 2009;284(31):21036-21046

20 Mori Y, Suzuma K, Uji A, et al. Restoration of foveal photoreceptors after intravitreal ranibizumab injections for diabetic macular edema. Sci Rep 2016;6(39161):39161

21 Decanini A, Karunadharma PR, Nordgaard CL, Feng X, Olsen TW, Ferrington DA. Human retinal pigment epithelium proteome changes in early diabetes. Diabetologia 2008;51(06):1051-1061
22 van Reyk DM, Gillies MC, Davies MJ. The retina: oxidative stress and diabetes. Redox Rep 2003;8(04):187-192

23 Sharma S, Saxena S, Srivastav K, et al. Nitric oxide and oxidative stress is associated with severity of diabetic retinopathy and retinal structural alterations. Clin Exp Ophthalmol 2015;43 (05):429-436

24 Hartnett ME, Lappas A, Darland D, McColm JR, Lovejoy S, D'Amore PA. Retinal pigment epithelium and endothelial cell interaction causes retinal pigment epithelial barrier dysfunction via a soluble VEGF-dependent mechanism. Exp Eye Res 2003;77 (05):593-599

25 Vlassara H, Cai W, Crandall J, et al. Inflammatory mediators are induced by dietary glycotoxins, a major risk factor for diabetic angiopathy. Proc Natl Acad Sci U S A 2002;99(24):15596-15601

26 Yamagishi S, Maeda S, Matsui T, Ueda S, Fukami K, Okuda S. Role of advanced glycation end products (AGEs) and oxidative stress in vascular complications in diabetes. Biochim Biophys Acta 2012; 1820(05):663-671

27 Sinha S, Saxena S, Prasad S, et al. Association of serum levels of anti-myeloperoxidase antibody with retinal photoreceptor ellipsoid zone disruption in diabetic retinopathy. J Diabetes Complications 2017;31(05):864-868

28 Nadri G, Saxena S, Kaur A, et al. Correlation between vitamin D serum levels and severity of diabetic retinopathy in patients with type 2 diabetes mellitus. Journal of Endocrinology, Metabolism and Diabetes of South Africa 2021;26(03):. Doi: 10.1080/16089677.2021.1903170

29 Ankita Stefanickova J, Saxena S, et al. Hyperglycemia potentiates the effect of ionic calcium in photoreceptor ellipsoid zone disruption in diabetic retinopathy. Int Ophthalmol 2019;39(10): 2237-2243

30 Maheshwary AS, Oster SF, Yuson RMS, Cheng L, Mojana F, Freeman WR. The association between percent disruption of the photoreceptor inner segment-outer segment junction and visual acuity in diabetic macular edema. Am J Ophthalmol 2010;150(01): 63-67.e1

31 Mishra N, Saxena S, Ruia S, et al. Increased levels of N( $(\varepsilon)$ - carboxy methyl lysine $(\mathrm{N}(\varepsilon)-\mathrm{CML})$ are associated with topographic alterations in retinal pigment epithelium: a preliminary study. J Diabetes Complications 2016;30(05):868-872

32 Achiron A, Kydyrbaeva A, Man V, et al. Photoreceptor integrity predicts response to anti-VEGF treatment. Ophthalmic Res 2017; 57(01):37-41

33 Chatziralli I, Theodossiadis G, Dimitriou E, Kazantzis D, Theodossiadis P. Association between the patterns of diabetic macular edema and photoreceptors' response after intravitreal ranibizumab treatment: a spectral-domain optical coherence tomography study. Int Ophthalmol 2020;40(10):2441-2448

34 De S, Saxena S, Kaur A, et al. Sequential restoration of external limiting membrane and ellipsoid zone after intravitreal antiVEGF therapy in diabetic macular oedema. Eye (Lond) 2021;35 (05):1490-1495

35 Jud P, Sourij H. Therapeutic options to reduce advanced glycation end products in patients with diabetes mellitus: a review. Diabetes Res Clin Pract 2019;148:54-63 